of MNNG may be accessible to the thiol at the active site. Given this, the chemoprotective activity of the glutathione $S$ transferase-mediated reaction of glutathione may be due to a denitrosation reaction that is predominant because the thiolate of GSH, when it reacts at carbon, generates an intermediate $\mathrm{T}^{-}$ that mainly reverts to starting materials due to the low conjugate acid $\mathrm{p} K_{\mathrm{a}}$ of the thiolate at the active site.

Supplementary Material Available: Tables S1-S4 of data from reactions of thiols with $N$-methyl- $N^{\prime}$-nitro- $N$-nitroguanidine, 40 ${ }^{\circ} \mathrm{C}$, ionic strength $1 \mathrm{M}(\mathrm{KCl})$ (14 pages). Ordering information is given on any current masthead page.

\title{
Coordination Isomerism in Pentamethylcyclopentadienyl-Substituted Iminophosphanes: From Classical Structures to a $\pi$-Complexed Iminophosphenium Ion
}

\author{
D. Gudat, ${ }^{\dagger}$ H. M. Schiffner, ${ }^{\dagger}$ M. Nieger,${ }^{\dagger}$ D. Stalke, ${ }^{\ddagger}$ A. J. Blake, ${ }^{\ddagger}$ H. Grondey,${ }^{\S}$ and \\ E. Niecke*, \\ Contribution from the Anorganisch Chemisches Institut der Universität, Universität Bonn, \\ Gerhard Domagk Strasse 1, D-5300 Bonn 1, Germany, Anorganisch Chemisches Institut der \\ Universität, Universität Göttingen, Tammannstrasse 4, D-3400 Göttingen, Germany, and Max \\ Planck Institut für Kohleforschung, Kaiser Wilhelm Platz 1, D-4330 Mühlheim/Ruhr, Germany. \\ Received October 16, 1991
}

\begin{abstract}
Novel iminophosphanes of the type $\mathrm{Me}_{5} \mathrm{C}_{5} \mathrm{P}=\mathrm{NR}\left(\mathrm{R}=\mathrm{C}_{6} \mathrm{H}_{2} \mathrm{tBu} \mathrm{u}_{3}(\mathbf{5 a}), \mathrm{Si}-i-\mathrm{Pr}_{3}(\mathbf{5 b}), \mathrm{SiMe}_{3}(\mathbf{5 c})\right)$ are prepared via either thermolytic or base-promoted elimination reactions and characterized by analytical and spectroscopic methods. The $X$-ray crystal structures of $\mathbf{5 a , b}$ establish the presence of coordination isomerism of the cyclopentadienyl ring, which at the same time strongly alters the characteristics of the P-N multiple bond. For $\mathbf{5 a}, \eta^{1}$-attachment of the $\mathrm{Me}_{5} \mathrm{C}_{5}$ moiety to the phosphorus and a localized phosphorus-carbon $\sigma$-bond are found, and the P-N double bond compares to that of alkylated iminophosphanes $\left(r_{\mathrm{PN}}=155.1(8) \mathrm{pm}\right)$. 5b exhibits $\eta^{2}$-coordination of the cyclopentadienyl ring leading to increased $\mathrm{P}-\mathrm{N}$ triple-bond character, as evidenced by shortening of the $\mathrm{P}-\mathrm{N}$ distance $(153.3(3) \mathrm{pm}$ ) and the remarkable opening of the nitrogen valence angle $\left(153.3(2)^{\circ}\right)$. The structure is discussed as an intramolecular $\pi$-complex between a formal cyclopentadienyl anion and an iminophosphenium cation. The ${ }^{1} \mathrm{H}$ and ${ }^{13} \mathrm{C}$ NMR spectra show that rapid elementotropic rearrangements around the five-membered ring take place both in solution and in the solid state. The unique temperature dependence of $\delta{ }^{31} \mathrm{P}$ in solution together with the considerable differences in $\delta^{31} \mathrm{P}$ between the solution and solid states are interpreted in a model assuming a "haptotropic" mechanism for the fluxionality involving dynamic $\eta^{1} / \eta^{2}$-coordination isomerizations in solution. As expected, nucleophilic displacement of the $\mathrm{Me}_{5} \mathrm{C}_{5}$ moiety takes place in the reaction of $5 \mathrm{~b}, \mathrm{c}$ with $\mathrm{LiC}_{6} \mathrm{H}_{2} \mathrm{tBu} \mathrm{Bu}_{3}$, yielding the iminophosphanes $\mathrm{tBu}_{3} \mathrm{H}_{2} \mathrm{C}_{6} \mathrm{P}=\mathrm{NR}\left(\mathrm{R}=\mathrm{Si}-i-\mathrm{Pr}_{3}(\mathbf{8 b}), \mathrm{SiMe}_{3}(\mathbf{8 c})\right)$ as products.
\end{abstract}

\section{Introduction}

Pentamethylcyclopentadienyl-substituted $\left(\mathrm{Me}_{5} \mathrm{C}_{5}\right)$ phosphorus-p $(\pi)$ multiple-bond systems are of both high synthetic and theoretical interest due to their unique stereoelectronic properties. While the $\mathrm{Me}_{5} \mathrm{C}_{5}$ group provides sufficient steric bulk for the stabilization of the low coordination number, ${ }^{1-3}$ its liability toward nucleophilic substitution ${ }^{3,4}$ at the same time permits a synthetically valuable functionalization of the double bond. In addition, the capacity for multihapto $\pi$-bonding ${ }^{5}$ leads to a versatility in structure and bonding. Thus, the cyclopentadienyls in the neutral systems $\mathrm{Me}_{5} \mathrm{C}_{5} \mathrm{P}=\mathrm{ER}_{n}\left(\mathrm{ER}_{n}=\mathrm{C}\left(\mathrm{SiMe}_{3}\right)_{2},{ }^{1} \mathrm{PC}_{5} \mathrm{Me}_{5}{ }^{3}\right)$ are $\eta^{1}$ $(\sigma)$-bonded in the solid state, while "nonclassical" $\eta^{2}(\pi)$-coordination prevails in the phosphenium cation $\left[\mathrm{Me}_{5} \mathrm{C}_{5} \mathrm{PN}(\mathrm{H}) \mathrm{tBu}\right]^{+6}$. In solution, all known systems are fluxional due to fast elementotropic rearrangements. ${ }^{6.7}$

Although an $\mathrm{Me}_{5} \mathrm{C}_{5}$-substituted iminophosphane has been prepared, ${ }^{2}$ no structure determinations of such compounds, which should constitute a "missing link" between $\eta^{1}-\mathrm{Me}_{5} \mathrm{C}_{5} \mathrm{P}=\mathrm{PR}$ and $\left[\eta^{2}-\mathrm{Me}_{5} \mathrm{C}_{5} \mathrm{PNHR}\right]^{+}$, have been performed yet. Here, we report the preparation and first structural characterization of novel $\mathrm{Me}_{5} \mathrm{C}_{5}$-substituted iminophosphanes. It is found that a substitution-induced coordination isomerism occurs between $\eta^{1}(\sigma)$ - and

\footnotetext{
Universităt Bonn.

Universität Göttingen.

Max Planck Institut für Kohleforschung.
}

$\eta^{2}(\pi)$-bonding of the $\mathrm{Me}_{5} \mathrm{C}_{5}$ ligand, which has most interesting implications on the structure and bonding of the phosphorusnitrogen multiple bond. The investigation of the dynamic aspects of the molecular structure was attempted by NMR spectroscopic studies of 5a both in the solid state and in solution, and evidence is presented that the dynamic process may follow different mechanisms in both cases.

\section{Experimental Section}

All manipulations were carried out under a dry argon atmosphere using standard Schlenk techniques. Solvents were dried by refluxing over appropriate reagents and distilled before use. $\mathrm{Me}_{5} \mathrm{C}_{5} \mathrm{Li}^{,}{ }^{8}$ (2,4,6-tritert-butylanilino)dichlorophosphane (2a), ${ }^{9}$ lithium bis(trimethylsilyl)amide (4), ${ }^{10}$ [bis(trimethylsilyl)amino]dichlorophosphane (6), ${ }^{11}$ and $\left(2,4,6\right.$-tri-tert-butylphenyl) lithium ${ }^{12}$ were prepared according to literature

(1) Gudat, D.; Niecke, E.; Krebs, B.; Dartmann, M. Chimia 1985, 39, 277.

(2) Gudat, D.; Niecke, E.; Krebs, B.; Dartmann, M. Organometallics 1986, 5,2376 .

(3) Jutzi, P.; Meyer, U.; Krebs, B.; Dartmann, M. Angew. Chem., Int. Ed. Engl. 1986, 25, 919

(4) Gudat, D.; Niecke, E. J. Chem. Soc. Chem. Commun. 1987, 10

(5) Jutzi, P. Adv. Organomet. Chem. 1986, 26, 217.

(6) Gudat, D.; Nieger, M.; Niecke, E. J. Chem. Soc., Dalton Trans. 1989, 693

(7) Jutzi, P. Chem. Rev. 1986, 86, 983

(8) Jutzi, P.; Saleske, H. Chem. Ber. 1984, 117, 222

(9) Niecke, E.; Lysek, M., Symalla, E. Chimia 1986, 40, 202.

(10) Wannagat, U.; Niederprüm, H. Chem. Ber. 1961, 94, 1540.

(11) Scherer, O. J.; Kuhn, N. J. Organomet. Chem. 1974, 82, C3. 
methods. (Triisopropylsilyl)amine (1b) was prepared as described in ref 13 and was purified by distillation (bp $43-45^{\circ} \mathrm{C} / 3$ Torr).

Solution ${ }^{31} \mathrm{P}\{\mathrm{l} \mathrm{H}\} \mathrm{NMR}$ spectra $(32.2 \mathrm{MHz})$ were recorded on a Varian FT80A, and ${ }^{1} \mathrm{H}$ (at 79.5 and $200 \mathrm{MHz}$ ) and ${ }^{13} \mathrm{C}\left({ }^{1} \mathrm{H}\right)$ NMR spectra (at 20.0 and $50.3 \mathrm{MHz}$ ) were recorded on both Varian FT80A and Bruker AC 200 instruments. For measurements at ambient temperature, $C_{6} D_{6}$ (previously dried over $4-\AA$ molecular sieves) was used as solvent unless otherwise noted. Chemical shifts are referenced to external TMS $\left({ }^{1} \mathrm{H}\right.$, ${ }^{13} \mathrm{C}$ ) or $85 \% \mathrm{H}_{3} \mathrm{PO}_{4}\left({ }^{31} \mathrm{P}\right)$; multiplicities refer to coupling with ${ }^{31} \mathrm{P}$ unless stated otherwise. Variable-temperature NMR spectra of $\mathbf{5 a , b}$ were obtained in toluene- $d_{8}\left({ }^{1} \mathrm{H},{ }^{13} \mathrm{C}\right)$ or hexane solution with approximately 0.15 $\mathrm{mL}$ of toluene- $d_{8}$ added in order to obtain a sufficient deuterium lock signal $\left({ }^{31} \mathrm{P}\right)$, respectively. Temperature measurements were performed by means of a ${ }^{31} \mathrm{P}$ chemical shift thermometer ${ }^{14}$ which was placed in a thin-walled 5-mm NMR tube and concentrically adjusted inside the 10-mm sample tube. Solid-state CP/MAS ${ }^{13} \mathrm{C}\left\{{ }^{1} \mathrm{H}\right\}$ and $\left.{ }^{31} \mathrm{P} \mid{ }^{1} \mathrm{H}\right\}$ spectra were recorded on a Bruker MSL 300 spectrometer under high-power proton decoupling. Spinning rates between 2500 and $3300 \mathrm{~Hz}$, relaxation delays of $4 \mathrm{~s} \mathrm{(} 3 \mathrm{~s}$ in ${ }^{31} \mathrm{P}$ spectra), and contact times of $1 \mathrm{~ms}$ were applied. The temperature was varied between 115 and $295 \mathrm{~K}$ with use of a temperature controller and nitrogen as the driving and bearing gas. Chemical shifts were referenced by using the methylene carbon resonance of adamantane $\left(\delta\left({ }^{13} \mathrm{C}\right) 38.4\right)$ or $\mathrm{H}_{3} \mathrm{PO}_{4}$ as external references. Loading of the rotors was carried out in an inert argon atmosphere using a specially designed apparatus as described elsewhere. ${ }^{15}$

Electron ionization $(70 \mathrm{eV})$ mass spectra were run on a VG 12-250 spectrometer. Elemental analyses were determined on a Heraeus $\mathrm{CHN}$ O-Rapid instrument; all melting points were determined in sealed glass capillaries.

Preparation of $\mathrm{PPr}_{3} \mathrm{SiNHPCl}_{2}$ (2b). To a stirred solution of the amine $1 \mathrm{~b}(0.115 \mathrm{~mol}, 30.0 \mathrm{~g})$ in hexanes $(50 \mathrm{~mL})$ was added dropwise 1 equiv of a $1.6 \mathrm{M}$ solution of $n-\mathrm{BuLi}$ in hexane $(0.115 \mathrm{~mol}, 72 \mathrm{~mL})$. The resulting mixture was stirred for $15 \mathrm{~min}$ and then added dropwise to a stirred solution of $\mathrm{PCl}_{3}(0.120 \mathrm{~mol}, 16.5 \mathrm{~g})$ in hexane $(100 \mathrm{~mL})$ at -78 ${ }^{\circ} \mathrm{C}$. After the reaction was warmed to ambient temperature, the precipitate was removed by filtration, and the solution was evaporated in vacuo. The residue was dissolved in pentane $(50 \mathrm{~mL})$ and cooled to -80 ${ }^{\circ} \mathrm{C}$. The product precipitated as white crystals, which were collected by filtration at low temperature and dried in vacuo: yield $87 \%(27.4 \mathrm{~g})$; $\mathrm{mp}$ $0^{\circ} \mathrm{C} ;{ }^{31} \mathrm{P}\left\{{ }^{\prime} \mathrm{H}\right\}$ NMR $\delta 168.3 ;$ ' $\mathrm{H}$ NMR $\delta 1.94\left(\mathrm{~m}, 21 \mathrm{H}, \mathrm{SiCH}\left(\mathrm{CH}_{3}\right)_{2}\right)$.

Preparation of $\mathrm{C}_{6} \mathrm{H}_{2} \mathrm{tBu}_{3} \mathrm{NHPClC}_{5} \mathrm{Me}_{5}$ (3a). A solution of compound 2a $(55.0 \mathrm{mmol}, 19.9 \mathrm{~g})$ in ether $(100 \mathrm{~mL})$ was added dropwise to a stirred, ice-cooled suspension of $\mathrm{Me}_{5} \mathrm{C}_{5} \mathrm{Li}(60.0 \mathrm{mmol}, 8.52 \mathrm{~g})$ in $120 \mathrm{~mL}$ of pentane. After the mixture was stirred for an additional $3 \mathrm{~h}$ at ambient temperature, the precipitated salts were removed by filtration, and the solution was evaporated in vacuo. The residue was dissolved in pentane and cooled to $-80^{\circ} \mathrm{C}$. The product separated as white crystals, which were collected by filtration and dried in vacuo: yield $72 \%(18.3$ g); mp $165^{\circ} \mathrm{C} ;{ }^{31} \mathrm{P}\left\{{ }^{1} \mathrm{H}\right\}$ NMR $\delta 153.0 ;{ }^{1} \mathrm{H}$ NMR $\delta 7.51\left(2 \mathrm{H}, \mathrm{C}_{6} \mathrm{H}_{2}\right), 1.92$ (broad, $15 \mathrm{H}, \mathrm{C}_{5}\left(\mathrm{CH}_{3}\right)_{5}, 1.53\left(18 \mathrm{H}, o-\mathrm{CCH}_{3}\right), 1.31\left(9 \mathrm{H}, p-\mathrm{CCH}_{3}\right)$.

Preparation of $\mathrm{iPr}_{3} \mathrm{SiNHPClC}_{5} \mathrm{Me}_{3}$ (3b). A solution of $2 \mathrm{~b}(55.0$ mmol, $15.0 \mathrm{~g})$ in pentane $(100 \mathrm{~mL})$ was reacted with $\mathrm{LiC}_{5} \mathrm{Me}_{5}$ in the same manner as that described for 3a. Crystallization at $-35^{\circ} \mathrm{C}$ yielded $81 \%(16.8 \mathrm{~g})$ of $3 \mathrm{~b}$ : $\mathrm{mp} 55^{\circ} \mathrm{C} ;{ }^{31} \mathrm{P}\left\{{ }^{1} \mathrm{H}\right\} \mathrm{NMR} \delta 148.6 ;{ }^{1} \mathrm{H}$ NMR $\delta 1.73$ $(3 \mathrm{H}), 1.56(3 \mathrm{H}), 1.50(3 \mathrm{H}), 1.33(\mathrm{~d}, 4.5 \mathrm{~Hz}, 3 \mathrm{H}), 1.16(\mathrm{~d}, 15 \mathrm{~Hz}$, $3 \mathrm{H}, \mathrm{C}_{5}\left(\mathrm{CH}_{3}\right)_{5}, 0.73\left(\mathrm{~m}, 21 \mathrm{H}, \mathrm{SiCH}\left(\mathrm{CH}_{3}\right)_{2}\right)$.

Preparation of $\mathrm{C}_{6} \mathrm{H}_{2} \mathrm{Bu}_{3} \mathrm{~N}=\mathrm{PC}_{5} \mathrm{Me}_{5}(5 \mathrm{Sa})$. A precooled $\left(-50^{\circ} \mathrm{C}\right)$ solution of $26.2 \mathrm{mmol}$ of lithium bis(trimethylsilyl)amide (4) $(4.40 \mathrm{~g})$ in THF $(30 \mathrm{~mL}$ ) was added slowly to a stirred solution of compound $3 \mathrm{a}$ $(26.2 \mathrm{mmol}, 12.2 \mathrm{~g})$ in THF $(40 \mathrm{~mL})$ at $-80^{\circ} \mathrm{C}$. When the addition was complete, the resulting yellow solution was gradually allowed to warm. Precipitation of $\mathrm{LiCl}$, which was accompanied by a deepening of the color, started above $-60^{\circ} \mathrm{C}$ and was complete at room temperature. After evaporation of the volatiles in vacuo, the residue was extracted with pentane $(100 \mathrm{~mL})$ and filtered. The filtrate was concentrated to $50 \mathrm{~mL}$ and cooled to $-30^{\circ} \mathrm{C}$. 5a precipitated as yellow crystals, which were collected by filtration and dried in vacuo: yield $63 \%(6.3 \mathrm{~g}) ; \mathrm{mp} 115^{\circ} \mathrm{C}$; MS $m / e 425(7), \mathrm{M}^{+}, 290\left(100, \mathrm{M}^{+}-\mathrm{C}_{5} \mathrm{Me}_{5}\right)$, and further fragmentation peaks; ${ }^{31} \mathrm{P}\left\{{ }^{1} \mathrm{H}\right\}$ NMR $\left(26^{\circ} \mathrm{C}\right.$, hexane $\left./ \mathrm{C}_{6} \mathrm{D}_{6}\right) \delta 194.9 ;{ }^{13} \mathrm{C}\left\{{ }^{1} \mathrm{H}\right\}$ NMR $\left(26^{\circ} \mathrm{C}\right) \delta 143.3(\mathrm{~d}, 8 \mathrm{~Hz}), 141.5,136.6(\mathrm{~d}, 7 \mathrm{~Hz}), 121.7\left(\mathrm{C}_{\mathrm{arom}}\right), 125.7$ (d, $\left.4 \mathrm{~Hz}, \mathrm{C}_{5}\right), 36.3,31.9$ (d, $\left.2 \mathrm{~Hz}, \mathrm{C}_{\mathrm{quart}}\right), 34.7,32.2\left(\mathrm{CCH}_{3}\right), 10.8$ $\left(\mathrm{C}_{5}\left(\mathrm{CH}_{3}\right)_{5}\right) ;{ }^{\prime} \mathrm{H}$ NMR $\left(26^{\circ} \mathrm{C}\right) \delta 7.60\left(2 \mathrm{H}, \mathrm{C}_{6} \mathrm{H}_{2}\right), 2.00\left(15 \mathrm{H}, \mathrm{C}_{5}\right.$. 3963

(12) Yoshifuji, M.; Shima, I.; Inamoto, N. Tetrahedron Lett. 1979, 21,

(13) Bowser, J. R.; Neilson, R. H.; Wells, R. L. Inorg. Chem. 1978, 17, 1882.

(14) Dickert, F. L.; Hellman, S. W. Anal. Chem. 1980, 52, 996.

(15) Benn, R.; Grondey, H.; Erker, G.; Aul, R.; Nolte, R. Organometallics $1990,9,2493$.
$\left.\left(\mathrm{CH}_{3}\right)_{5}\right), 1.68\left(18 \mathrm{H}, o-\mathrm{CCH}_{3}\right), 1.46\left(9 \mathrm{H}, p-\mathrm{CCH}_{3}\right)$. Anal. Calcd for $\mathrm{C}_{28} \mathrm{H}_{44} \mathrm{NP}$ : C, 79.01; $\mathrm{H}, 10.42 ; \mathrm{N}, 3.29$. Found: $\mathrm{C}, 76.83 ; \mathrm{H}, 10.47$; N, 3.51.

Preparation of $\mathrm{iPr}_{3} \mathrm{SiN}=\mathrm{PC}_{5} \mathrm{Me}_{5}$ (5b). Compound $5 \mathrm{~b}$ was prepared in the same manner as that described for $5 \mathrm{a}$ from $3 \mathrm{~b}$ ( $26.2 \mathrm{mmol}, 9.80$ g) and $4(26.2 \mathrm{mmol}, 4.40 \mathrm{~g})$. The crude product was purified by vacuum distillation: bp $85^{\circ} \mathrm{C}(0.01$ Torr); yield $70 \%(6.10 \mathrm{~g})$; MS m/e 337 (23, $\left.\mathrm{M}^{+}\right), 294\left(97, \mathrm{M}^{+}-\mathrm{iPr}\right), 202\left(23, \mathrm{M}^{+}-\mathrm{C}_{5} \mathrm{Me}_{5}\right), 160\left(100, \mathrm{M}^{+}-\mathrm{iPr}\right.$ $\left.-\mathrm{C}_{5} \mathrm{Me}_{5}\right)$, and further fragmentation peaks; ${ }^{31} \mathrm{P}\left\{{ }^{1} \mathrm{H}\right\}$ NMR $\left(26^{\circ} \mathrm{C}\right.$ hexane $\left./ \mathrm{C}_{6} \mathrm{D}_{6}\right) \delta 159$ (broad); ${ }^{13} \mathrm{C}\left({ }^{1} \mathrm{H}\right\}$ NMR $\left(26^{\circ} \mathrm{C}\right) \delta 124.5(\mathrm{~d}, 5 \mathrm{~Hz}$, $\left.\mathrm{C}_{5}\right), 18.7\left(\mathrm{SiCCH}_{3}\right), 13.3(\mathrm{~d}, 1 \mathrm{~Hz}, \mathrm{SiC}), 10.2\left(\mathrm{~d}, 2 \mathrm{~Hz}, \mathrm{C}_{5}\left(\mathrm{CH}_{3}\right)_{5}\right) ;{ }^{1} \mathrm{H}$ NMR $\left(26^{\circ} \mathrm{C}\right) \delta 1.80\left(\mathrm{~d}, 0.6 \mathrm{~Hz}, 15 \mathrm{H}, \mathrm{C}_{5}\left(\mathrm{CH}_{3}\right)_{5}\right), 1.14(18 \mathrm{H})$ and 1.01 $(3 \mathrm{H}), \mathrm{AB}_{6} \mathrm{X}$ spin system, $J_{\mathrm{AB}}=7.5 \mathrm{~Hz}, J_{\mathrm{AX}}=0.5 \mathrm{~Hz}, J_{\mathrm{BX}}=0$, $\mathrm{SiCH}^{\mathrm{A}}\left(\mathrm{CH}^{\mathrm{B}}{ }_{3}\right)_{2}\left(\mathrm{X}={ }^{31} \mathrm{P}\right)$. Anal. Calcd for $\mathrm{C}_{19} \mathrm{H}_{36} \mathrm{NPSi}$ : C, $67.61 ; \mathrm{H}$, $10.75 ; \mathrm{N}, 4.15$. Found: $\mathrm{C}, 65.14 ; \mathrm{H}, 10.89 ; \mathrm{N}, 4.59$.

Preparation of $\mathrm{Me}_{3} \mathrm{SiN}=\mathrm{PC}_{3} \mathrm{Me}_{3}(5 \mathrm{c})$. Reaction of $6(100 \mathrm{mmol}$, $26.2 \mathrm{~g})$ with $\mathrm{LiC}_{5} \mathrm{Me}_{5}(110 \mathrm{mmol})$ in pentane $(200 \mathrm{~mL})$ in the same manner as that described for $3 a$ afforded the chlorophosphane 7 . The crude product was transferred into a distillation apparatus that was equipped with a 10-cm Vigreux column and a distillation head and was refluxed at $120^{\circ} \mathrm{C}$ in vacuo. $\mathrm{Me}_{3} \mathrm{SiCl}$, which formed during the elimination, was collected in a cold trap $\left(-196^{\circ} \mathrm{C}\right)$. After the reaction was complete (approximately $1 \mathrm{~h}$ ), the iminophosphane that formed was distilled off at the same temperature. The product was purified by fractionated redistillation to yield pure $5 c$ as a deep yellow liquid of bp $52-55^{\circ} \mathrm{C}$ (0.01 Torr): yield $75 \%$ (18.9 g); MS $\mathrm{m} / \mathrm{e} 253\left(34, \mathrm{M}^{+}\right), 135$ $\left(79, \mathrm{C}_{5} \mathrm{Me}_{5}{ }^{+}\right), 118\left(100, \mathrm{M}^{+}-\mathrm{C}_{5} \mathrm{Me}_{5}\right), 73\left(82, \mathrm{Me}_{3} \mathrm{Si}^{+}\right)$, and further fragmentation peaks; $\left.{ }^{31} \mathrm{P} \mid{ }^{l} \mathrm{H}\right\}$ NMR $\left(26^{\circ} \mathrm{C}\right.$, hexane $\left./ \mathrm{C}_{6} \mathrm{D}_{6}\right) \delta 138$ (broad); ${ }^{13} \mathrm{C}\left({ }^{1} \mathrm{H}\right\} \mathrm{NMR}\left(26^{\circ} \mathrm{C}\right) \delta 124.1\left(\mathrm{~d}, 4 \mathrm{~Hz}, \mathrm{C}_{5}\right), 10.2\left(\mathrm{~d}, 3 \mathrm{~Hz}, \mathrm{C}_{5}\left(\mathrm{CH}_{3}\right)_{5}\right)$, $3.9(\mathrm{~d}, 2 \mathrm{~Hz}, \mathrm{SiC}) ;{ }^{1} \mathrm{H}$ NMR $\left(26^{\circ} \mathrm{C}\right) \delta 1.80\left(15 \mathrm{H}, \mathrm{C}_{5}\left(\mathrm{CH}_{3}\right)_{5}\right), 0.31(9$ $\mathrm{H}, \mathrm{SiCH}_{3}$ )

Preparation of $\mathrm{iPr}_{3} \mathrm{SiN}=\mathrm{PC}_{6} \mathrm{H}_{2} \mathrm{tBu}_{3}(10 \mathrm{~b})$. A solution of compound 5 b $(4.20 \mathrm{mmol}, 1.40 \mathrm{~g})$ in THF ( $3 \mathrm{~mL}$ ) was rapidly added via syringe to a stirred solution (2,4,6-tri-tert-butylphenyl)lithium $(4.20 \mathrm{mmol}, 1.06$ $\mathrm{g})$ in THF $(10 \mathrm{~mL})$ at $-80^{\circ} \mathrm{C}$. The reaction mixture was allowed to warm to ambient temperature over $1 \mathrm{~h}$. After removal of the solvent in vacuo, the residue was extracted with pentane $(25 \mathrm{~mL})$ and filtered. The filtrate was evaporated and the residue distilled in vacuo to afford $10 \mathrm{~b}$ as a deep yellow liquid of bp $135-138^{\circ} \mathrm{C}(0.01$ Torr $)$, which solidified below room temperature: yield $53 \%(1.0 \mathrm{~g}) ; \mathrm{mp} 10-15^{\circ} \mathrm{C} ;{ }^{31} \mathrm{P}\left\{{ }^{1} \mathrm{H}\right\}$ NMR $\left(26{ }^{\circ} \mathrm{C}\right.$, hexane $\left./ \mathrm{C}_{6} \mathrm{D}_{6}\right) \delta 497.5 ;{ }^{13} \mathrm{C}\left\{{ }^{1} \mathrm{H}\right\}$ NMR $\delta 154.6(\mathrm{~d}, 102 \mathrm{~Hz}), 153.3$ $(\mathrm{d}, 6 \mathrm{~Hz}), 150.7,122.2\left(\mathrm{C}_{\mathrm{grom}}\right), 38.5,35.1\left(\mathrm{CCH}_{3}\right), 34.8(\mathrm{~d}, 7 \mathrm{~Hz}), 31.4$ $\left(\mathrm{C}_{\text {quart }}\right), 18.8\left(\mathrm{SiCCH}_{3}\right), 13.3(\mathrm{SiC}){ }^{1} \mathrm{H}$ NMR $87.50\left(2 \mathrm{H}, \mathrm{C}_{6} \mathrm{H}_{2}\right), 1.60$ $\left(9 \mathrm{H}, p-\mathrm{CCH}_{3}\right), 1.30\left(18 \mathrm{H}, o-\mathrm{CCH}_{3}\right), 1.2\left(\mathrm{~m}, 21 \mathrm{H}, \mathrm{SiCH}\left(\mathrm{CH}_{3}\right)_{2}\right)$. Anal. Calcd for $\mathrm{C}_{28} \mathrm{H}_{44} \mathrm{NP}$ : $\mathrm{C}, 72.43 ; \mathrm{H}, 11.25 ; \mathrm{N}, 3.13$. Found: $\mathrm{C}$, $71.17 ; \mathrm{H}, 11.48 ; \mathrm{N}, 1.63$.

X-ray Crystal Structure Determination of Sa,b. The diffraction data were collected with Nicolet R3m (5a) and STOE AED (5b) diffractometers, respectively. In the case of $5 \mathbf{b}$ a semiempirical absorption correction on the basis of $\Psi$-scans was applied $\left(T_{\min / \max }=0.500 / 0.623\right)$. The structures were solved by direct methods. All non-hydrogen atoms were refined anisotropically. $\mathrm{H}$-atoms were located by difference electron density determinations and were either refined by using a riding model with isotropic thermal parameters fixed at 1.2 times that of the bonded carbon (5a) or refined free with common isotropic thermal parameters (5b). The $\mathrm{C}-\mathrm{H}$ distance was fixed at $0.96 \AA$ in both cases. Calculations were performed with SHELXTL-Plus (Sheldrick, G. M. SHELXTLPlus, Siemens Aralytical X-ray Instruments Inc.: Madison, WI, 1989).

Details on the structure solution are given in Table I. The atomic coordinates and isotropic temperature factors are given in Tables II (5a) and III (5b), respectively. Further details of the crystal structure determinations are available on request from the Fachinformationszentrum Karlsruhe, Gesellschaft für wissenschaftlich-technische Information $\mathrm{mbH}, \mathrm{D}-7514$ Eggenstein-Leopoldshafen 2, Germany, on quoting the depository number CSD-55060, the names of the authors, and the journal citation.

\section{Results and Discussion}

Synthesis and Characterization. The synthesis of the iminophosphanes 5a,b followed a known procedure for the generation of $\mathrm{P}=\mathrm{N}$ double bonds via metalation and subsequent salt elimination from aminohalophosphanes. ${ }^{16}$ The necessary $\mathrm{Me}_{5} \mathrm{C}_{5^{-}}$ substituted precursors $3 \mathbf{a}, \mathrm{b}$ were prepared from $\mathrm{PCl}_{3}$ and the primary amines $1 \mathbf{a}, \mathbf{b}$ in two steps according to standard procedures (Scheme I). Treatment of THF solutions of $3 a, b$ with the lithium amide 4 at $-78^{\circ} \mathrm{C}$ ( 1 equiv) and warming to ambient temperature then afforded the iminophosphanes 5a,b (Scheme I). Pure

(16) Niecke, E.; Gudat, D. Angew. Chem., Int. Ed. Engl. 1991, 30, 217. 
Scheme I

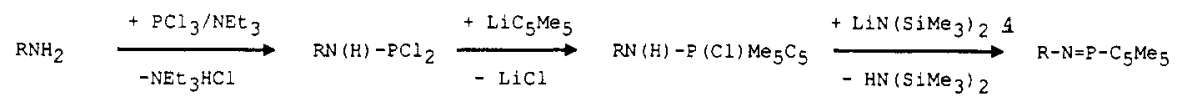

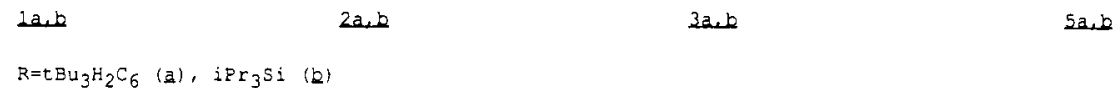

Scheme II

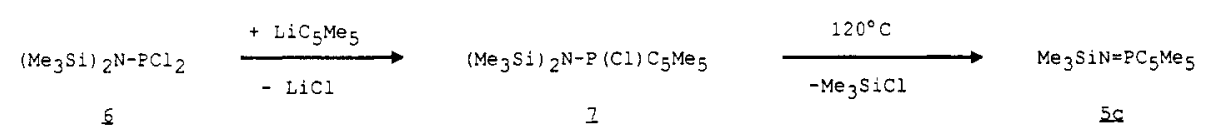

Table I. Crystallographic Data and Summary of Data Collection

\begin{tabular}{|c|c|c|}
\hline & $5 \mathbf{a}$ & $5 \mathbf{b}$ \\
\hline formula & $\mathrm{C}_{28} \mathrm{H}_{44} \mathrm{NP}$ & $\mathrm{C}_{19} \mathrm{H}_{36} \mathrm{NPSi}$ \\
\hline cryst syst & monoclinic & monoclinic \\
\hline space group & $P 2_{1} / n$ (No. 14) & $P 2_{1} / n$ (No. 14 ) \\
\hline $\begin{array}{l}\text { cryst dimens, mm } \\
\text { cryst color }\end{array}$ & $\begin{array}{l}0.2 \times 0.2 \times 0.4 \\
\text { orange }\end{array}$ & $\begin{array}{l}0.55 \times 0.6 \times 0.7 \\
\text { yellow }\end{array}$ \\
\hline temp, K & & 120 \\
\hline$a, \AA$ & $11.974(6)$ & $8.466(2)$ \\
\hline$b, \AA$ & $13.436(4)$ & $11.397(2)$ \\
\hline$c, \AA$ & $17.309(5)$ & $22.082(5)$ \\
\hline$\beta, \operatorname{deg}$ & $95.80(3)$ & $98.75(2)$ \\
\hline$V, \AA^{3}$ & 2770 & 2106 \\
\hline $\boldsymbol{Z}$ & 4 & 4 \\
\hline$\rho_{\text {calod }}, \mathrm{g} \mathrm{cm}^{-3}$ & 1.02 & 1.07 \\
\hline$\mu, \mathrm{mm}^{1}$ & 0.11 & 0.18 \\
\hline$F(000)$ & 936 & 744 \\
\hline $\max 2 \theta, \operatorname{deg}$ & 45 & 50 \\
\hline no. of variables & 271 & 216 \\
\hline no. of reflens coll & 3950 & 3901 \\
\hline no. of unique data & 3611 & 3695 \\
\hline$R$ (merge) & 0.023 & 0.119 \\
\hline $\begin{array}{l}\text { no. of data used in refinement } \\
\qquad|F|>4 \sigma(F)\end{array}$ & 1320 & 3398 \\
\hline $\boldsymbol{R}$ & 0.079 & 0.064 \\
\hline $\boldsymbol{R}_{\mathrm{w}}$ & 0.066 & 0.075 \\
\hline & 0.0003 & 0.0001 \\
\hline $\begin{array}{l}\text { largest feature in final } \\
\text { diff map, } e / \AA^{3}\end{array}$ & 0.32 & 0.87 (near $P$ ) \\
\hline
\end{tabular}
and Refinement ${ }^{a, b}$

${ }^{a}$ All data were taken with graphite-monochromated Mo $\mathrm{K} \alpha$ radiation with $\lambda=0.71073 \AA$ by using the $\bar{\omega}$-scan (5a) and profile fitting technique $^{b}$ (5b), respectively. $R=\sum \| F_{\mathrm{o}}|-| F_{\mathrm{c}}|/| \sum\left|F_{\mathrm{o}}\right|$ and $R_{\mathrm{w}}=\sum$. $\left(\left|F_{\mathrm{o}}\right|-\left|F_{\mathrm{c}}\right|\right) w^{1 / 2} / \Sigma\left|F_{\mathrm{o}}\right| w^{1 / 2}$ with weighting scheme $w^{-1}=\sigma^{2}(F)+g F^{2}$ ${ }^{\circ}$ Clegg, W. Acta Crystallogr. 1981, A37, 22-28.

products were isolated after filtration, removal of volatiles in vacuo, and recrystallization from pentane (5a) or distillation (5b) of the residue. $5 \mathbf{a}$ may be alternatively obtained in similar yields via treatment of the iminophosphane, $\mathrm{ClP}=\mathrm{N}\left(\mathrm{C}_{6} \mathrm{H}_{2} \mathrm{tBu}\right)_{3}$, with $\mathrm{Me}_{5} \mathrm{C}_{5} \mathrm{Li}^{17}$

According to a different route, the trimethylsilylated derivative $5 c$ was accessible via thermolytic $\left(120^{\circ} \mathrm{C}\right)$ elimination of $\mathrm{Me}_{3} \mathrm{SiCl}^{16}$ from the chlorophosphane 7 , which was prepared in situ by treatment of $\left(\mathrm{Me}_{3} \mathrm{Si}\right)_{2} \mathrm{NPCl}_{2}(6)$ with 1 equiv of $\mathrm{Me}_{5} \mathrm{C}_{5} \mathrm{Li}$ (Scheme II). The product was collected by distillation and was redistilled for purification.

Identification and characterization of $5 \mathrm{a}-\mathrm{c}$ were established from their mass spectra and analytical and multinuclear $\left({ }^{1} \mathrm{H},{ }^{13} \mathrm{C}\right.$, ${ }^{31} \mathrm{P}$ ) NMR data. 5a,b were further characterized by $\mathrm{X}$-ray crystallography.

$\mathbf{X}$-ray Structural Investigations. Suitable single crystals of $\mathbf{5 a , b}$ were obtained by recrystallization from pentane at low temperature. Although $\mathbf{5 c}$ was also found to solidify upon cooling below $-30^{\circ} \mathrm{C}$, no sufficient single crystals could be obtained in this case. Due to the low melting point (approximately $-30^{\circ} \mathrm{C}$ ) of $5 \mathrm{~b}$, the structure determination also had to be performed at low temperature.

(17) Reichert, F. Ph.D. Thesis, University of Bonn, Germany, 1989.
Table II. Atomic Coordinates $\left(\times 10^{4}\right)$ and Equivalent Isotropic

\begin{tabular}{|c|c|c|c|c|}
\hline & $x$ & $y$ & $z$ & $U(\mathrm{eq})^{a}$ \\
\hline$P(1)$ & $295(2)$ & $1209(2)$ & $2481(2)$ & 49 (1) \\
\hline$N(1)$ & $-704(6)$ & $1947(5)$ & 2459 (4) & $44(3)$ \\
\hline$C(1)$ & $-353(9)$ & $-111(7)$ & $2513(9)$ & $53(5)$ \\
\hline$C(2)$ & 410 (13) & $-412(9)$ & $3173(8)$ & $68(7)$ \\
\hline$C(3)$ & $1379(12)$ & $-740(8)$ & $2900(12)$ & $80(7)$ \\
\hline$C(4)$ & $1241(14)$ & $-741(9)$ & 2084 (12) & $81(7)$ \\
\hline $\mathrm{C}(5)$ & 213 (13) & $-415(8)$ & $1835(8)$ & $65(6)$ \\
\hline$C(6)$ & $-1614(8)$ & $-164(7)$ & $2516(8)$ & $104(6)$ \\
\hline$C(7)$ & 147 (14) & $-365(10)$ & $4001(8)$ & 171 (11) \\
\hline$C(8)$ & $2424(10)$ & $-1043(9)$ & 3399 (10) & 194 (11) \\
\hline$C(9)$ & $2113(11)$ & $-1071(10)$ & $1562(10)$ & 206 (11) \\
\hline$C(10)$ & $-370(13)$ & $-361(9)$ & $1010(7)$ & $143(9)$ \\
\hline$C(11)$ & $-627(7)$ & $3011(7)$ & $2449(7)$ & $41(4)$ \\
\hline$C(12)$ & $-527(8)$ & $3514(7)$ & $3171(7)$ & $45(5)$ \\
\hline$C(13)$ & $-302(7)$ & $4550(8)$ & $3152(7)$ & $49(5)$ \\
\hline$C(14)$ & $-236(8)$ & $5075(7)$ & $2475(8)$ & $40(4)$ \\
\hline$C(15)$ & -487 (7) & 4559 (7) & $1796(6)$ & $40(5)$ \\
\hline$C(16)$ & $-712(8)$ & $3535(7)$ & $1739(6)$ & $43(5)$ \\
\hline$C(17)$ & $-628(11)$ & $3039(8)$ & $3966(6)$ & $62(5)$ \\
\hline$C(18)$ & $-831(11)$ & $3822(8)$ & $4591(6)$ & $130(7)$ \\
\hline$C(19)$ & $434(9)$ & $2468(9)$ & $4253(6)$ & $94(6)$ \\
\hline$C(20)$ & $-1652(8)$ & $2354(8)$ & $3942(6)$ & $83(5)$ \\
\hline$C(21)$ & $68(9)$ & $6178(8)$ & 2498 (9) & $67(6)$ \\
\hline$C(22)$ & $-697(13)$ & $6752(9)$ & $2931(10)$ & 187 (11) \\
\hline$C(23)$ & $-46(17)$ & $6644(9)$ & $1711(9)$ & $206(13)$ \\
\hline$C(24)$ & 1216 (11) & $6321(8)$ & $2817(12)$ & $239(15)$ \\
\hline$C(25)$ & $-1026(9)$ & $3058(8)$ & $949(6)$ & $53(5)$ \\
\hline$C(26)$ & $-1395(9)$ & $3847(8)$ & $318(6)$ & $96(6)$ \\
\hline$C(27)$ & $-2019(8)$ & $2344(8)$ & $943(6)$ & $82(5)$ \\
\hline$C(28)$ & $3(9)$ & $2512(8)$ & $669(6)$ & $89(6)$ \\
\hline
\end{tabular}
Displacement Parameters $\left(\AA^{2} \times 10^{3}\right)$ of $\mathbf{5 a}$

${ }^{a}$ Equivalent isotropic $U$ defined as one-third of the trace of the orthogonalized $\mathbf{U}_{i j}$ tensor.

Table III. Atomic Coordinates $\left(\times 10^{4}\right)$ and Equivalent Isotropic Displacement Parameters $\left(\AA^{2} \times 10^{3}\right)$ of $5 \mathrm{~b}$

\begin{tabular}{lrrrr}
\hline & \multicolumn{1}{c}{$\boldsymbol{x}$} & $y$ & \multicolumn{1}{c}{$\boldsymbol{z}$} & $U(\mathrm{eq})^{a}$ \\
\hline $\mathrm{P}$ & $286(1)$ & $3226(1)$ & $2015(1)$ & $21(1)$ \\
$\mathrm{Si}$ & $1926(1)$ & $2732(1)$ & $850(1)$ & $20(1)$ \\
$\mathrm{N}$ & $1404(4)$ & $3182(3)$ & $1531(1)$ & $26(1)$ \\
$\mathrm{C}(1)$ & $136(4)$ & $5027(3)$ & $2319(2)$ & $23(1)$ \\
$\mathrm{C}(2)$ & $1292(4)$ & $4365(3)$ & $2732(2)$ & $25(1)$ \\
$\mathrm{C}(3)$ & $450(5)$ & $3825(3)$ & $3171(2)$ & $28(1)$ \\
$\mathrm{C}(4)$ & $-1173(4)$ & $4102(3)$ & $3024(2)$ & $25(1)$ \\
$\mathrm{C}(5)$ & $-1384(4)$ & $4829(3)$ & $2503(2)$ & $23(1)$ \\
$\mathrm{C}(1 \mathrm{M})$ & $548(5)$ & $5894(3)$ & $1860(2)$ & $34(1)$ \\
$\mathrm{C}(2 \mathrm{M})$ & $3062(4)$ & $4501(4)$ & $2762(2)$ & $36(1)$ \\
$\mathrm{C}(3 \mathrm{M})$ & $1172(6)$ & $3051(4)$ & $3694(2)$ & $46(2)$ \\
$\mathrm{C}(4 \mathrm{M})$ & $-2508(5)$ & $3640(4)$ & $3336(2)$ & $42(2)$ \\
$\mathrm{C}(5 \mathrm{M})$ & $-2944(5)$ & $5325(4)$ & $2189(2)$ & $40(1)$ \\
$\mathrm{C}(11)$ & $3716(4)$ & $1732(3)$ & $1038(2)$ & $28(1)$ \\
$\mathrm{C}(12)$ & $4301(6)$ & $1209(4)$ & $477(2)$ & $51(2)$ \\
$\mathrm{C}(13)$ & $5124(5)$ & $2269(4)$ & $1469(2)$ & $37(1)$ \\
$\mathrm{C}(21)$ & $2270(4)$ & $4094(3)$ & $390(2)$ & $25(1)$ \\
$\mathrm{C}(22)$ & $2336(6)$ & $3853(4)$ & $-286(2)$ & $41(1)$ \\
$\mathrm{C}(23)$ & $3714(5)$ & $4827(3)$ & $676(2)$ & $30(1)$ \\
$\mathrm{C}(31)$ & $196(5)$ & $1890(3)$ & $403(2)$ & $29(1)$ \\
$\mathrm{C}(32)$ & $-217(5)$ & $774(3)$ & $738(2)$ & $38(1)$ \\
$\mathrm{C}(33)$ & $-1275(5)$ & $2661(4)$ & $253(2)$ & $38(1)$ \\
\hline
\end{tabular}

${ }^{a}$ Equivalent isotropic $U$ defined as one-third of the trace of the orthogonalized $\mathbf{U}_{i j}$ tensor. 


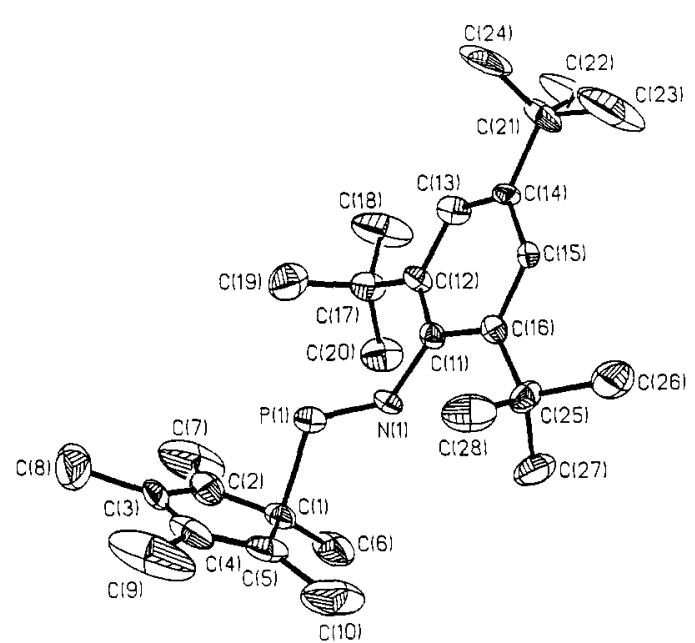

Figure 1. Molecular structure of 5a. Selected bond distances (pm) and angles (deg): $\mathrm{P}(1)-\mathrm{N}(1) 155.1$ (8), $\mathrm{P}(1)-\mathrm{C}(1) 194.0$ (10), $\mathrm{C}(1)-\mathrm{C}(2)$ 144.7 (19), C(2)-C(3) 136.9 (23), C(3)-C(4) 140.5 (29), C(4)-C(5) 133.7 (22), C(1)-C(5) 147.0 (21); C(1)-P(1)-N(1) 106.0 (4), P(1)-N(1) $-\mathrm{C}(11) 125.9$ (6).

In crystalline $\mathbf{5 a}$ the $\eta^{\prime}$-coordination of the $\mathrm{Me}_{5} \mathrm{C}_{5}$ ligand is clearly evident (Figure 1 ). The $E$ (trans) $\mathrm{P}-\mathrm{N}$ double-bond distance $(155 \mathrm{pm})$ and the $\mathrm{C} 1-\mathrm{P}-\mathrm{N}$ angle $\left(106^{\circ}\right)$ lie in the expected ranges for trans-iminophosphanes, ${ }^{16}$ and the bond data of the $\mathrm{P}=\mathrm{NC}_{6} \mathrm{H}_{2} \mathrm{tBu}$ moiety are very similar to those of comparable compounds. ${ }^{16}$ While the planarity of $\mathrm{C} 2-\mathrm{C5}$ (within experimental error) and the alternate $\mathrm{C}-\mathrm{C}$ bond distances in the five-membered ring agree with a description as a cyclic diene bearing the phosphorus substituent in the allylic position, ${ }^{18}$ the allylic carbon exhibits severe distortions from ideal tetrahedral geometry. The $\mathrm{P}-\mathrm{C} 1-\mathrm{C} 2\left(93^{\circ}\right)$ and $\mathrm{P}-\mathrm{C} 1-\mathrm{C} 5\left(91^{\circ}\right)$ bond angles are distinctly contracted and the $\mathrm{C} 2-\mathrm{C} 1-\mathrm{C} 6$ (methyl) and $\mathrm{C} 5-\mathrm{C} 1-\mathrm{C} 6$ angles widened $\left(122^{\circ}\right)$ as compared to the expected angle of $109.4^{\circ}$. At the same time the $\mathrm{Cl}-\mathrm{P}$ distance $(194 \mathrm{pm})$ is considerably longer than a normal $\mathrm{P}-\mathrm{C}$ single bond, ${ }^{19}$ corresponding to a very low Pauling bond order (PBO) ${ }^{20}$ of 0.76 . The observed geometry indicates a state between ideal $\mathrm{sp}^{3}$ and $\mathrm{sp}^{2}$ hybridization at $\mathrm{Cl}$, which implies a low degree of s-electron density in the $\mathrm{Cl}-\mathrm{P}$ bond. Characteristic bond deformations of the same type, although somewhat less pronounced as for $5 \mathrm{a}$, have previously been reported for other $\eta^{1}-\mathrm{Me}_{5} \mathrm{C}_{5}$-substituted phosphorus $p(\pi)$-systems, ${ }^{1,3}$ demonstrating that bonding is only marginally different in all of these cases.

In contrast to the situation described above, 5b (Figure 2) exhibits a slightly asymmetric $\eta^{2}$-attachment of the planar fivemembered ring (the standard deviation for the best plane fitted through $\mathrm{Cl}-\mathrm{C} 5$ is $\sigma=0.87 \mathrm{pm}$ ) and represents the first example of $\mathrm{Me}_{5} \mathrm{C}_{5}$ multihapto bonding in a neutral phosphorus $\mathrm{p}(\pi)$-system. Four out of five bond distances in the five-membered ring lie in a narrow range between 139 and 143 pm with PBOs between 1.30 (C1-C5) and $1.52(\mathrm{C} 3-\mathrm{C} 4)$. The P-bridged $\mathrm{C} 1-\mathrm{C} 2$ bond is slightly longer (144.4 pm, PBO 1.21), so that a marked equalization of bond lengths results as compared to 5a. Of the ring carbons, C3-C5 are planar within experimental error, and the sum of $\mathrm{C}-\mathrm{C}-\mathrm{C}$ angles at $\mathrm{C} 1\left(359 \pm 1^{\circ}\right)$ and $\mathrm{C} 2\left(358 \pm 1^{\circ}\right)$ indicates that deviations of the adjacent methyl groups out of the ring plane are negligible. Hence, $s^{2}$ hybridization may be assumed for all five ring atoms. In the $\mathrm{PNSiR}_{3}$ fragment, rather short distances are found for both the $\mathrm{Si}-\mathrm{N}$ bond $(171 \mathrm{pm})$ and the $\mathrm{P}-\mathrm{N}$ double bond $(153.3 \mathrm{pm})$, which is shorter than the $\mathrm{P}-\mathrm{N}$ bond in $5 \mathrm{a}$ by $1.8 \mathrm{pm}$. Together with the large $\mathrm{P}-\mathrm{N}-\mathrm{Si}$ angle $\left(153^{\circ}\right)$, this suggests a state between pure $\mathrm{sp}$ and $\mathrm{sp}^{2}$ hybridization

(18) Lee, T. J.; Schaefer, H. F., III; Magnusson, E. A. J. Am. Chem. Soc. $1985,107,7239$.

(19) Corbridge, D. E. C. The Structural Chemistry of Phosphorus; Elsevier: Amsterdam, 1974.

(20) Pauling, L. The Nature of the Chemical Bond, 3rd. ed.; Cornell University Press: Ithaca, NY, 1960; pp 239 ff.

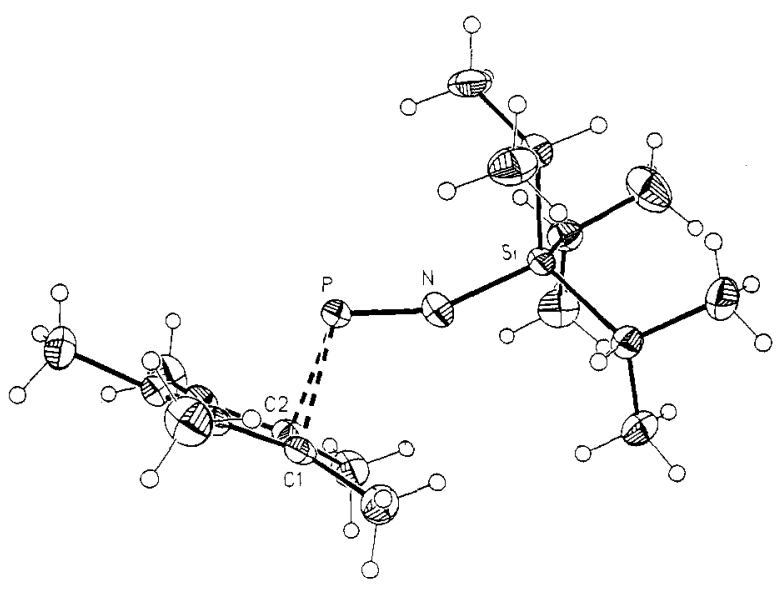

Figure 2. Molecular structure of $5 \mathrm{~b}$. Selected bond distances (pm) and angles (deg): P-N 153.3 (3), P-C(1) 216.8 (4), P-C(2) 212.2 (4), $\mathrm{C}(1)-\mathrm{C}(2) 144.5(5), \mathrm{C}(2)-\mathrm{C}(3) 142.8$ (5), C(3)-C(4) 139.9 (5), C. (4)-C(5) $140.8(5), \mathrm{C}(1)-\mathrm{C}(5) 142.5(5), \mathrm{Si}-\mathrm{N} 170.9$ (3); P-N-Si 153.3 (2).

for the two-coordinate nitrogen, with the P-N bond adopting partial triple-bond character. $A \mathrm{Me}_{5} \mathrm{C}_{5}-\mathrm{P}$ interaction that is much weaker than in $\left[\eta^{2}-\mathrm{Me}_{5} \mathrm{C}_{5} \mathrm{PNHtBu}\right]^{+}(8)^{6}$ is evidenced by the long $\mathrm{P}-\mathrm{Cl}(212 \mathrm{pm})$ and $\mathrm{P}-\mathrm{C} 2$ distances $(217 \mathrm{pm})$, which correspond to extremely low PBOs of 0.32 and 0.38 , respectively, that are presumably the longest $\mathrm{P}-\mathrm{C}$ bonds known. Even if the angle between the cyclopentadienyl ring and the plane through $\mathrm{P}, \mathrm{Cl}$, and $\mathrm{C} 2\left(86.6^{\circ}\right)$ is smaller than in $8\left(90.7^{\circ}\right)$, the P-C3/-C4 distances $(263 / 264 \mathrm{pm})$ indicate that bonding between these atoms may be neglected.

The $\eta^{2}-\mathrm{Me}_{5} \mathrm{C}_{5}-$ phosphorus bonding situation described here is a common feature of cyclopentadienyl $\pi$-complexes of main group elements. ${ }^{5}$ Very similar structural parameters have been found for $\eta^{2}-\mathrm{Me}_{5} \mathrm{C}_{5}$-substituted germylenes ${ }^{21}$ and the phosphenium cation 8. ${ }^{6}$ An analogous geometry was also calculated for an $\eta^{2}$-cyclopentadienyl in $\left(\mathrm{C}_{5} \mathrm{H}_{5}\right)_{2} \mathrm{P}^{+} .18$ The longer $\mathrm{P}-\mathrm{Cl} /-\mathrm{C} 2$ distances and the more pronounced leveling of the ring bonds in the neutral $\mathbf{5 b}$ indicate, however, that bonding between the $\mathrm{Me}_{5} \mathrm{C}_{5}$ and $\mathrm{PN}$ fragments is considerably weaker than in the cationic 8 . In principle, the electronic structure of $\mathbf{5 b}$ can be explained as an intramolecular donor/acceptor $\pi$-complex between a cyclopentadienyl anion and an iminophosphenium cation ${ }^{22}$ with a formal P-N triple bond. Stabilization of an iminophosphenium ion in an intramolecular $\sigma$-complex has recently been proposed for the compound $\left[\mathrm{tBu}_{2} \mathrm{PSe}_{2}\right]\left[\mathrm{PNC}_{6} \mathrm{H}_{2} \mathrm{tBu}_{3}\right]$ (9). ${ }^{23}$ The $\eta^{2}$-bonding situation in $\mathbf{5 b}$ is in accord with both the low electrophilic character of the nitrogen in PN triple-bond systems ${ }^{22}$ as well as the tendency of phosphorus to adopt a low formal coordination number in its $\pi$-complexes. $6,7,18$

NMR Spectroscopic Investigations. The solution ${ }^{1} \mathrm{H}$ and ${ }^{13} \mathrm{C}$ NMR spectra of $\mathbf{5 a , b}$ exhibit sharp average signals for all ring carbons and methyl groups of the $\mathrm{Me}_{5} \mathrm{C}_{5}$ moiety down to the lowest accessible temperatures of $-90^{\circ} \mathrm{C}$. Such behavior had previously been reported as a common feature of compounds $\mathrm{Me}_{5} \mathrm{C}_{5} \mathrm{P}=\mathrm{ER}_{n}{ }^{1-3}$ and was attributed to an elementotropic migration of the phosphorus fragment around the ring occurring with a very low activation energy. ${ }^{7}$ Since the activation barriers for exchange are frequently higher in the solid state than in solution, ${ }^{24}$ the ${ }^{13} \mathrm{C}-\mathrm{CP} / \mathrm{MAS}$ spectra of solid $5 \mathrm{a}$ were studied in the temperature range between 20 and $-158^{\circ} \mathrm{C}$. However, as in solution, the spectra lack any dynamically induced broadening or splitting for the resonances of the $\mathrm{Me}_{5} \mathrm{C}_{5}$ moiety (Figure 3), thus demonstrating that the fluxional behavior still persists in the solid

(21) Jutzi, P.; Hampel, B.; Hursthouse, M. B.; Howes, A. J. Organometallics 1986, $5,1944$.

(22) Niecke, E.; Nieger, M.; Reichert, F. Angew. Chem., Int. Ed. Engl. 1988, 27, 1715 .

(23) Niecke, E.; Nieger, M.; Reichert, F.; Schoeller, W. W. Angew. Chem. Int. Ed. Engl. 1988, 27, 1713.

(24) Examples for this are cited in ref 15. 


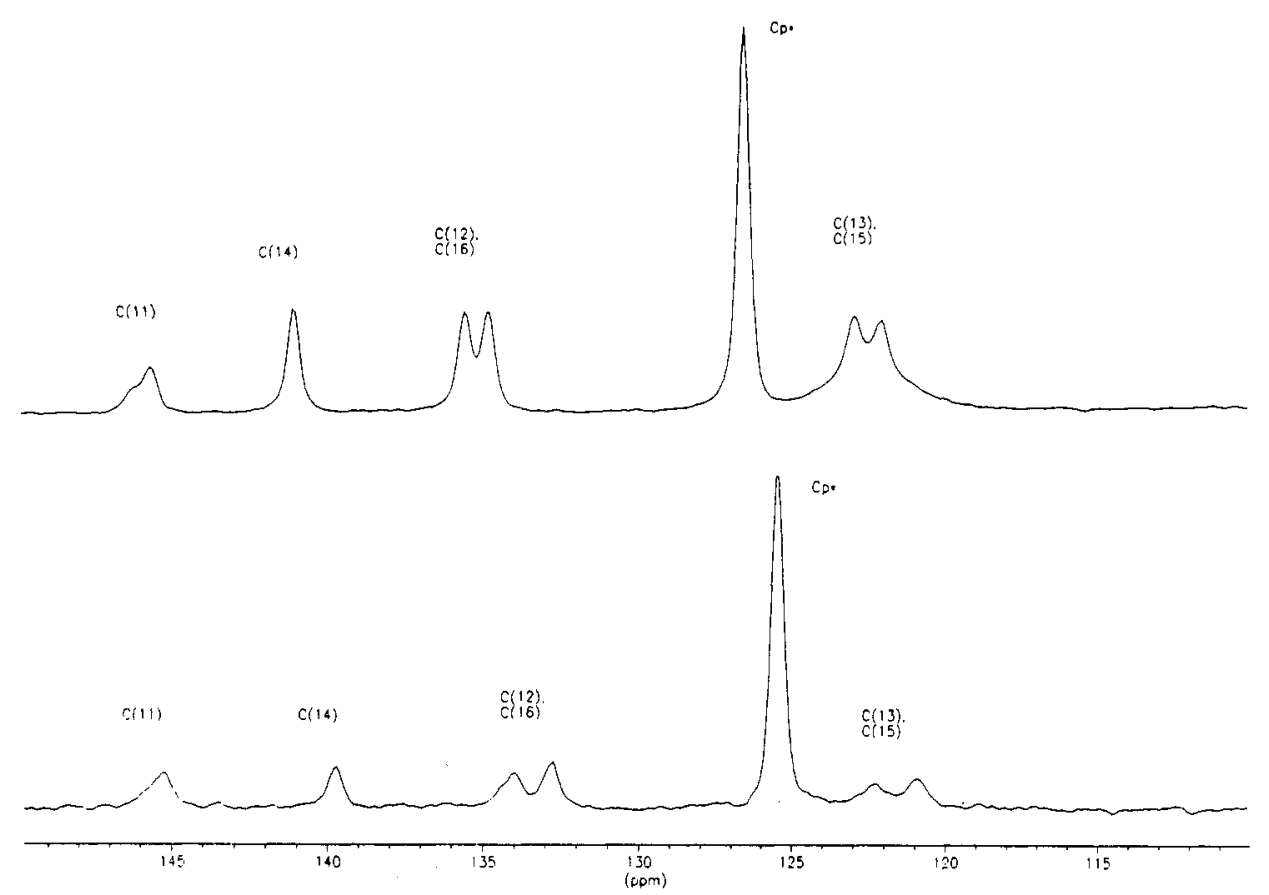

Figure 3. Olefinic region (center band) of the CP/MAS- ${ }^{13} \mathrm{C}\left({ }^{1} \mathrm{H}\right\} \mathrm{NMR}$ spectrum at $20^{\circ} \mathrm{C}$ (top) and $-158^{\circ} \mathrm{C}$ (bottom). The intense signal marked $\mathrm{Cp}^{*}$ is assigned to the five carbons of the cyclopentadienyl ring. The resonances of the aromatic carbons are marked as $\mathrm{C}(11)-\mathrm{C}(16)$ in accord with the numbering scheme in Figure 1. Due the low symmetry of the crystal, the equivalence of the ortho and meta positions of the aryl ring is removed, and separate resonances are observed for $\mathrm{C}(12), \mathrm{C}(16)$ and $\mathrm{C}(13), \mathrm{C}(15)$, respectively.

state. ${ }^{25}$ Even if no mechanistic information is extractable from the NMR spectra, the X-ray structures of 5a,b give strong evidence that the rotational motion proceeds by a sequence of 1,2 shifts, which is in accord with the general interpretation of the cyclopentadienyl dynamics as $[1,5]$-sigmatropic rearrangements. ${ }^{7}$

In regard to the similarity of the solution and solid-state ${ }^{13} \mathrm{C}$ spectra of $5 \mathbf{a}$, the significant differences in the ${ }^{31} \mathrm{P}$ spectra were quite unexpected (see Figure 4). In the solid, $\delta^{31} \mathrm{P}$ (iso) 269.9 ppm at $25^{\circ} \mathrm{C}$ was shifted slightly downfield ( $\Delta \delta 8 \mathrm{ppm}$ ) upon cooling to $-158^{\circ} \mathrm{C}$, which is sufficiently explained as the consequence of thermal expansion effects. In solution, a much stronger deshielding of the signal occurred between 25 and -90 ${ }^{\circ} \mathrm{C}(\Delta \delta 42 \mathrm{ppm})$; moreover, at $25^{\circ} \mathrm{C}, \delta{ }^{31} \mathrm{P}$ differed by $75 \mathrm{ppm}$ between the solid state and hexane solution $\left(\delta^{31} \mathrm{P} 195 \mathrm{ppm}\right)$. Temperature- or medium-induced chemical shift variations of this magnitude are unprecedented for low-coordinate phosphorus compounds. ${ }^{26}$ An explanation of the effects becomes feasible, postulating a nonsigmatropic mechanism for the ring migration for the solution case, which involves dynamic exchange between discernible $\eta^{1}$ - and $\eta^{2}$-coordination isomers and is in analogy to the $\eta^{1}-/ \eta^{2}-\mathrm{Me}_{5} \mathrm{C}_{5}$ coordination isomerism in solid 5a,b. Due to the fast exchange rate, the observed chemical shift should represent the population weighted average whose variation reflects the temperature-induced changes of individual populations. Support for this model is gained from the fact that the observed temperature dependence of $\delta{ }^{31} \mathrm{P}$ can be excellently fitted to an appropriate theoretical expression, ${ }^{27}$ with the calculated shift of the $\eta^{1}$ isomer in very good agreement with $\delta^{31} \mathrm{P}($ iso) in the solid state (see Figure 4). A "haptotropic" two-step mechanism for the cyclopentadienyl migration had been suggested earlier on kinetic grounds for the complexes of $\mathrm{Me}_{5} \mathrm{C}_{5}$-substituted acyls with $\mathrm{AlCl}_{3}{ }^{28}$

(25) A significant broadening which is observed at low temperature for the signals of the primary carbons of the $\mathrm{C}_{6} \mathrm{H}_{2}(\mathrm{tBu})_{3}$ moiety may be assigned to slowing of the rotation of the tBu groups in the solid state.

(26) Measurements of $\delta^{31} \mathrm{P}$ for a number of $\mathrm{P}$ alkyl-, amino-, and halogeno-substituted derivatives showed that in all cases $\delta^{31} \mathrm{P}$ remained constant within $4 \mathrm{ppm}$ between ambient temperature and $-80^{\circ} \mathrm{C}$ : Gudat, D. Ph.D. Thesis, University of Bielefeld, Germany, 1987.

(27) Dalling, D. K.; Zilm, K. W.; Grant, D. M.; Heeschen, W. A.; Horton, W. I. J. Am. Chem. Soc. 1981, 103, 4817.

(28) Childs, R. F.; Zeya, M. J. Am. Chem. Soc. 1974, 96, 6418. Childs, R. F. Tetrahedron 1982, 38, 567 .

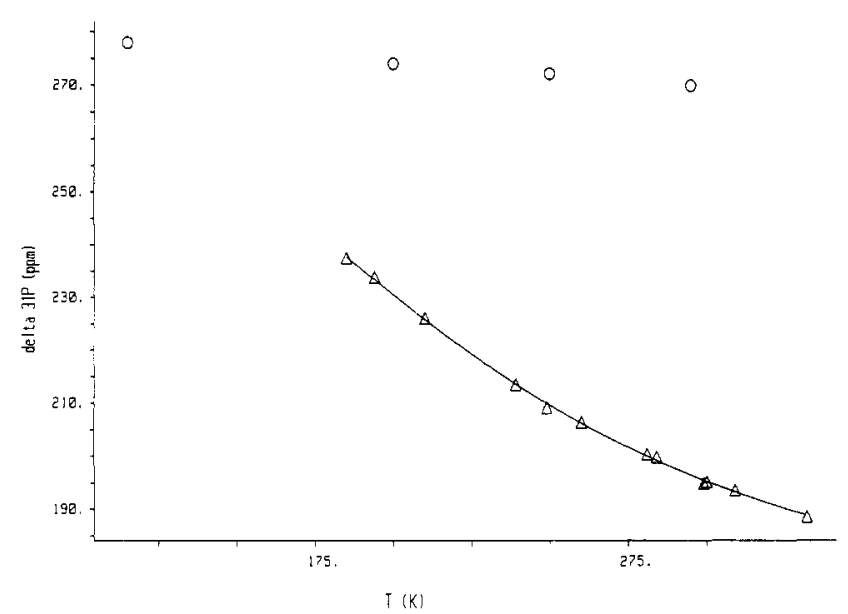

Figure 4. Temperature dependence of $\delta^{31} \mathrm{P}$ of $5 \mathrm{a}$ in solution $(\Delta)$ and the solid state $(0)$. The straight line shows the results (with standard deviations) of a fit of the observed ${ }^{31} \mathrm{P}$ chemical shifts (in ppm) to a functional expression according to a two-site-exchange model ${ }^{27}(T$ in $\mathrm{K})$, $\delta^{\text {obsod }}=\left[\delta\left(\eta^{1}\right.\right.$ isomer $)+\delta\left(\eta^{1}\right.$ isomer $\left.) e^{(\Delta S / R)} e^{(\Delta H / R T)}\right] /\left[1+e^{(\Delta S / R)} e^{(\Delta H / R T)}\right]$, with $\delta\left(\eta^{1}\right.$ isomer $)=268(30) \mathrm{ppm}, \delta\left(\eta^{1}\right.$ isomer $)=149(20) \mathrm{ppm}, \Delta S=$ $S\left(\eta^{1}\right.$ isomer $)-S\left(\eta^{2}\right.$ isomer $)=24(8) \mathrm{J} \mathrm{K}^{-1} \mathrm{~mol}^{-1}, \Delta H=\Delta H\left(\eta^{1}\right.$ isomer $)$ $-\Delta H\left(\eta^{2}\right.$ isomer $)=-6.1(1.3) \mathrm{kJ} \mathrm{mol}^{-1}$.

Since in that case the high electrophilicity of the migrating double-bond system was considered vital for both the stabilization of the $\eta^{2}$ isomer and the observed depression of the activation barrier, ${ }^{28}$ the electrophilic nature of PN multiple bonds ${ }^{16}$ presents an argument in favor of the proposed model. It must be noted, however, that the available experimental data in this case provide a rather indirect clue and that alternative mechanisms of the dynamics (e.g., cis/trans isomerization of the double bond) cannot be ruled out.

In contrast to $\mathbf{5 a}$, the solution spectra of $\mathbf{5 b}$ exhibit only weak deshielding of $\delta^{31} \mathrm{P}$ with increasing temperature; however, a marked change of the line shape becomes evident: the singlet observable at low temperatures eventually broadens and above ambient temperature splits into a triplet. The 1:1:1 intensity distribution and different line widths for the inner and outer lines are characteristic features of coupling with the ${ }^{14} \mathrm{~N}$ nucleus ${ }^{29}$ 
Scheme III

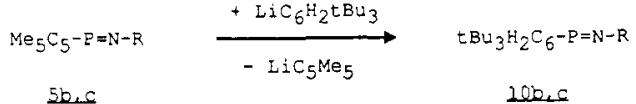

$$
\begin{aligned}
& R=\operatorname{sil}_{3} \text { (b) } \operatorname{SiMe}_{3}(c)
\end{aligned}
$$

$\left({ }^{1} J^{14} \mathrm{~N}^{31} \mathrm{P}=62 \mathrm{~Hz}\right)$. The appearance of this coupling, which is to our knowledge unprecedented in the chemistry of P-N compounds, indicates a near-linear geometry or very rapidly inverting structure for the two-coordinate nitrogen which may be correlated with the partial P-N triple-bond character in the solid state.

Nucleophilic Substitution Reactions. $\eta^{1}-\mathrm{Me}_{5} \mathrm{C}_{5}$-substituted two-coordinate phosphorus compounds are known to undergo displacement of the $\mathrm{Me}_{5} \mathrm{C}_{5}$ group very easily by organic or organometallic nucleophiles. ${ }^{1,3,4,16}$ In regard to the extremely weak $\mathrm{Me}_{5} \mathrm{C}_{5}-\mathrm{P}$ bonding in $\mathbf{5 b}$, similar behavior is expected for the corresponding $\eta^{2}-\mathrm{Me}_{5} \mathrm{C}_{5}$ isomers.

As a test of this hypothesis, the reactions of the $\mathrm{N}$-silylated compounds $5 b, c$ with the organic nucleophile, $\mathrm{LiC}_{6} \mathrm{H}_{2} \mathrm{tBu} \mathrm{Bu}_{3}{ }^{12}$ were investigated. In both cases, treatment of the iminophosphane with an equimolar amount of the organolithium reagent afforded the corresponding substitution products $10 \mathrm{~b}, \mathrm{c}$ in almost quantitative yield (as monitored by ${ }^{31} \mathrm{P}$ NMR). Whereas $10 \mathrm{~b}$ was identified by comparison of its spectroscopic data with an authentic sample, 10c was isolated in good yield after distillative workup and characterized by analytical and spectroscopic methods. Further investigations of the substitution chemistry of $5 a-c$ including other organic and organometallic nucleophiles are currently under way (Scheme III).

Conclusions

The synthesis and structural characterization of the iminophosphanes $\mathbf{5 a}$ and $\mathbf{5 b}$ as stable $\mathrm{P}-\mathrm{N}$ double-bond systems con-

(29) Pople, J. A. Mol. Phys. 1958, 1, 168

(30) Romanenko, V. D.; Ruban, A. V.; Markovskii, L. N. J. Chem. Soc., Chem. Commun. 1983, 187. taining discernible $\eta^{1}$ - or $\eta^{2}-\mathrm{Me}_{5} \mathrm{C}_{5}$ ligands verify the coordinational isomerism which had been postulated for these compounds. The results of the $\mathrm{X}$-ray structures suggest localized $\mathrm{Me}_{5} \mathrm{C}_{5}-\mathrm{P} \sigma$ bonding in 5a, whereas $5 \mathrm{~b}$ can be described as a cyclopentadienyl $\pi$-complex of a formally triple-bonded aminophosphenium ion. The existence of coordination isomerism further demonstrates that both $\sigma$ - and $\pi$-bonded species are very close in energy, as had been predicted on the basis of calculations for cyclopentadienylaminophosphenium ions. ${ }^{31}$ The preference for either configuration can be controlled by differential effects as the variation of substituents, the promotion of the $\eta^{2}$-coordination in $5 b$ being consistent with an increased electrophilicity of the P-N multiple bond as a consequence of the silyl substitution. ${ }^{32}$

The ${ }^{13} \mathrm{C}-\mathrm{CP} / \mathrm{MAS}$ spectra of $5 \mathrm{a}$ prove that the fluxionality of the $\mathrm{Me}_{5} \mathrm{C}_{5}$ ligand persists at low temperature in the solid state. Further, comparison of the solution and solid-state ${ }^{31} \mathrm{P}$ spectra gives evidence that the molecular structure in solution may differ from that in the crystal, which is tentatively explained by a model assuming a "haptotropic" mechanism involving dynamic $\eta^{1}-/$ $\eta^{2}$-coordination isomerizations for the cyclopentadienyl migration in solution.

In accord with the low $\eta^{2}-\mathrm{Me}_{5} \mathrm{C}_{5}-\mathrm{P}$ bond order is the chemical reactivity of $\mathbf{5 b}, \mathbf{c}$, which allows nucleophilic substitution of the cyclopentadienyl with preservation of the double-bond system. Hence, $\mathrm{Me}_{5} \mathrm{C}_{5}$-substituted iminophosphanes can be regarded as promising synthons for novel P-functionalized iminophosphanes or iminophosphenium ions.

Acknowledgment. We thank the Deutsche Forschungsgemeinschaft (SFB 334) and the Fonds der Chemischen Industrie for financial support.

(31) Baxter, S. G.; Cowley, A. H.; Mehrotia, S. K. J. Am. Chem. Soc. $1981,103,5572$.

(32) (a) Gudat, D.; Niecke, E.; Schoeller, W. W.; Rademacher, P. J. Chem. Soc., Chem. Common. 1985, 1050. (b) Gudat, D.; Niecke, E.; Sachs, W.; Rademacher, P. Z. Anorg. Allg. Chem. 1987, 545, 7. 